\title{
Perioden mit Smileys. \\ Zum Verhältnis von Emoticons und Interpunktion*
}

Karsten Rinas und Veronika Uhrová (Olomouc)

\begin{abstract}
This study deals with smileys/emoticons which are frequently used in e-mails, chats and other modern types of text. The use of these signs is analyzed from the viewpoint of the theory of writing; special attention is paid to the question of the relationship of these signs to (German) punctuation. For this purpose, smileys and traditional punctuation are compared under various aspects (emotionality, irony, structuring of text). Another component of this study is a discussion of reflections on the use of smileys found in the pertinent literature and in internet forums. It is shown that the "system of smileys" shares several common features with the older theory of punctuation and can therefore be regarded as a (rudimentary) system of punctuation in a nascent state.
\end{abstract}

\section{$1 \quad$ Einleitung}

Gegenstand dieses Beitrags sind die sogenannten Smileys, also jene ,aus einzelnen ASCIIZeichen zusammengesetzten Bilder, die als menschliche Gesichter interpretiert werden können und in E-Mails oder in Chats eingesetzt werden, um den Gemütszustand des Schreibers anzudeuten [...] oder Ironie zu kennzeichnen” (Klussmann 1999, s. v. Smileys). Als prototypisches und wohl am weitesten verbreitetes Smiley (,Ur-Smiley“) kann die Zeichenkombination :-) angesehen werden, welche ,ein auf der Seite liegendes lachendes Gesicht” darstellt (ebd.). ${ }^{1}$ Smileys werden auch mit dem durch Kontamination/Blending (aus Emotion und Icon) gebildeten Wort Emoticon bezeichnet (vgl. etwa Klussmann 1999, s. v. Emoticon; Ghersi/Lee/Karadagi 2002: 75; Sjurts 2006: 53).

Seit den 1980er Jahren werden Smileys in wachsendem Maße in der elektronischen Kommunikation verwendet; sie haben sich „zu einem festen Bestandteil der Internetkommunikation verfestigt" (Schmidt 2000: 124). Das Inventar dieser Zeichen ist jedoch bis heute nicht konsolidiert (vgl. etwa Haase et al. 1997: 64; Hess-Lüttich/Wilde 2003: 174). Neben dem prototypischen Smiley :-) sind diverse weitere Kombinationen in Gebrauch.

\footnotetext{
* Dieser Beitrag wurde gefördert durch das Projekt „Studie k raným dějinám německé interpunkce” (IGA UP FF_2013_043) der Palacký-Universität Olomouc. Für kritische Anmerkungen bedanken wir uns bei Marie Krappmann, Veronika Opletalová und den anonymen Gutachtern von Linguistik online.

${ }^{1}$ Dies dürfte auch der historisch früheste Smiley sein. Dieser wurde angeblich zuerst von Scott Fahlman in den frühen 1980er Jahren - zunächst an der Carnegie Mellon University in einem Mailbox-System - kreiert; vgl. ELL2, Bd. 9, s. v. Pictography, 595; Haase et al. (1997: 64), Lischka (2007). 
In dem Verzeichnis The Unofficial Smiley Dictionary werden u. a. die folgenden „Basic Smileys" aufgelistet und erläutert:

\begin{tabular}{|l|l|}
\hline$:-)$ & $\begin{array}{l}\text { Your basic smiley. This smiley is used to inflect a sarcastic or joking statement } \\
\text { since we can't hearvoice inflection over e-mail. }\end{array}$ \\
\hline$;-)$ & $\begin{array}{l}\text { Winky smiley. User just made a flirtatious and/or sarcastic remark. More of a } \\
\text { "don't hit me for what I just said" smiley. }\end{array}$ \\
\hline$:-($ & $\begin{array}{l}\text { Frowning smiley. User did not like that last statement or is upset or depressed } \\
\text { about something. }\end{array}$ \\
\hline$:-$ I & Indifferent smiley. Better than a [ :-( ] but not quite as good as a [ :-) ]. \\
\hline$:->$ & User just made a really biting sarcastic remark. Worse than a [ ;-) ]. \\
\hline$>:->$ & User just made a really devilish remark. \\
\hline$>;->$ & Winky and devil combined. A very lewd remark was just made. \\
\hline
\end{tabular}

Tabelle 1: Grundlegende Smileys nach The Unofficial Smiley Dictionary

In diesen Funktionsbeschreibungen werden die Smileys mit Gefühlszuständen sowie der Markierung von Ironie/Sarkasmus korreliert. Darüber hinaus ist das Bemühen erkennbar, eine Hierarchie der Smileys zu bestimmen.

Diese Liste ist jedoch weder erschöpfend noch allgemein verbindlich. Vielmehr kursieren im Internet diverse Entwürfe solcher Smiley-Systeme, ${ }^{2}$ wobei die ,genaue Bedeutung” dieser Smileys „nirgendwo offiziell spezifiziert ist” (Klussmann 1999, s. v. Smileys), was erwartungsgemäß zu Schwankungen führt. Beispielsweise werden in der whatis? com Encyclopedia (2002: 234f.) unter anderem folgende Zeichenkombinationen aufgeführt und definiert:

\begin{tabular}{|l|l|}
\hline$:-)$ & Smile \\
\hline$;-)$ & Smile with a wink \\
\hline$:-($ & Sad \\
\hline$:-$ I & Grim \\
\hline
\end{tabular}

Tabelle 2: Zeichenkombinationen nach whatis?com

Während die Kombination :-I also nach The Unofficial Smiley Dictionary Indifferenz zum Ausdruck bringt, signalisiert sie der whatis?com Encyclopedia zufolge Grimmigkeit. ${ }^{3}$

Ein weiteres Beispiel: Nach Wirth (2005: 81), ,ist das Smiley :-) ein Zeichen für gute Laune, das iterierte Smiley :-))) bezeichnet sehr gute Laune und das winky Smiley ;-) dient als Ironie-

\footnotetext{
2 Vgl. Sanderson (1993, 1997). Vgl. etwa auch die Auflistungen in Haase et al. (1997: 83) und Runkehl, Schlobinski und Siever (1998: 64), Crystal (2006: 40). Es gibt sogar spezielle „Insider-Smileys“ für einzelne Newsgroups (vgl. Runkehl/Schlobinski/Siever 1998: 70).

${ }^{3}$ In analoger Weise reicht auch die Interpretationsskala für die entsprechende graphische Gesichtsdarstellung $\odot$ „,von Gleichgültigkeit über Frustration bis hin zu Grießgrämigkeit”; vgl. die Web-Seite Einfügen von Smileys.
} 
signal”. Nach The Unofficial Smiley Dictionary dient aber auch das Ur-Smiley :-) zur Markierung von Sarkasmus/Ironie.

Unabhängig von derlei Schwankungen lässt sich konstatieren, dass die gängigen Smileys grundsätzlich mit Bedeutungen/Funktionen, nicht aber mit fixen phonemsprachlichen Repräsentationen verknüpft sind. Dies legt es nahe, sie zu den Piktogrammen zu rechnen (vgl. Kjørup 1997: 3504; ELL2, Bd. 9, s. v. Pictography). Mitunter werden sie auch als Ideogramme klassifiziert (vgl. etwa Haase et al. 1997: 63-65; ELL2, Bd. 4, s. v. E-Mail), was insofern nicht verwundert, als die Abgrenzung von Piktogrammen und Ideogrammen generell unscharf ist (vgl. etwa Dürscheid 2012: 64). Auf jeden Fall kann der Ausdruck von Emotionen an stilisierten Gesichtern als ikonisch gewertet werden (vgl. etwa Beißwenger 2000: 97; ELL2, Bd. 4, s. v. E-Mail), so dass Smileys sich der Großgruppe der ikonischen Zeichen zuordnen lassen. Es wäre somit möglich und sinnvoll, sie innerhalb der Semiotik zu behandeln (vgl. Kjørup 1997; Trautsch/Wu 2012). Ob sie auch im Rahmen der Linguistik untersucht werden sollten, ist nicht so eindeutig entscheidbar. Als Piktogramme ließen sich die Smileys dem Vorbereich der Schrift zuordnen (vgl. etwa Dürscheid 2012: 64-66; vgl. auch Lutz 1996), was eine Behandlung im Rahmen der Schriftlinguistik möglich, aber nicht zwingend erscheinen lässt.

Dieser etwas unklare und periphere Status der Smileys spiegelt sich auch in der Literatur wider. In den linguistischen Fachlexika von Bussmann (ed.) (2002) und Glück (ed.) (2010) sind Smileys/Emoticons nicht behandelt; in der großen Encyclopedia of Language \& Linguistics (ELL2) sind sie ebenfalls nicht als Stichwort aufgeführt, doch werden sie zumindest sporadisch behandelt, am ausführlichsten im Beitrag zur Piktographie (Bd. 9: 595). Ergiebigere Nachschlagewerke in Bezug auf unser Thema sind Speziallexika zum Bereich Internet / neue Medien (vgl. etwa Klussmann 1999, s. v. Smileys; Ghersi/Lee/Karadagi 2002: 75; whatis?com Encyclopedia 2002: 234; Ferretti 2004: 251; Sjurts 2006: 53).

Auch die allgemeineren Darstellungen zur Schriftlinguistik widmen den Smileys wenig oder gar keine Aufmerksamkeit. In dem grundlegenden Sammelband von Günther und Ludwig (eds.) $(1994,1996)$ werden sie ebenso wenig behandelt wie in der Darstellung von Dürscheid (2012). Von den linguistischen Beiträgen sind jene am ergiebigsten, die der sprachlichen Kommunikation im Internet gewidmet sind. ${ }^{4}$ Hier wird das Phänomen der Smileys sogar oft besonders herausgehoben. Dies gilt etwa für das Werk von Crystal (2006), wo die Sprache des Internets durchgehend als „Netspeak“ bezeichnet und als ,,a genuine language variety” (2006: 98) betrachtet wird. Und gerade die Smileys werden von Crystal als ,one of the most distinctive features” des „Netspeak” gewertet (2006: 42). Es gibt jedoch auch Forscher, die die Auffälligkeiten des „Net-Jargons“ oder „Cyberslangs“ nicht in den Rang einer eigenen Varietät befördern wollen, sondern statt dessen dafür plädieren, diese Charakteristika durch die Besonderheiten der Internet-Kommunikation zu erklären (vgl. etwa ELL2, Bd. 7, s. v. Media; Schlobinski 2001; Dürscheid 2003).

\footnotetext{
4 Vgl. etwa Weingarten (ed.) (1997), Runkehl, Schlobinski und Siever (1998), Thimm (ed.) (2000), Siever, Schlobinski und Runkehl (eds.) (2005) und Crystal (2006). Auch hier scheint das Interesse allerdings nachgelassen zu haben. Das Verhältnis von Smileys und Internetkommunikation wurde offenbar um die Jahrtausendwende am intensivsten diskutiert.
} 
Die Termini Smiley und Emoticon werden oft synonym gebraucht. Mitunter wird jedoch eine Unterscheidung getroffen, wonach als Emoticons die mit Schriftzeichen dargestellten (,liegenden") Darstellungen von Gesichtern bestimmt werden (wie in allen bisherigen Beispielen), während unter Smileys grafische Gesichtsdarstellungen wie etwa :) oder :) verstanden werden (vgl. etwa Wikipedia: Smiley und Emoticon). Wir werden diese Unterscheidung hier nicht streng durchführen, doch werden wir noch auf die Frage zu sprechen kommen, ob diese Differenzierung in linguistischer Hinsicht sinnvoll ist.

Das Hauptziel dieses Beitrags ist eine Charakterisierung des Smiley-Gebrauchs aus schriftlinguistischer Perspektive. Insbesondere soll hier die Frage diskutiert werden, in welchem Verhältnis Smileys zum (deutschen) Interpunktionssystem stehen. Können Smileys als eine spezielle Art von Interpunktionszeichen gedeutet werden ${ }^{5}$ Welche Eigenschaften verbinden die Smileys mit den Interpunktionszeichen? Wo gibt es Unterschiede?

Im Hinblick auf die bisher referierte Charakterisierung der Smileys/Emoticons liegt es nahe, deren Verhältnis zur Interpunktion im Ausgang von zwei Eigenschaften zu bestimmen, die für sie als charakteristisch gelten: der Ausdruck von Gefühlen und die Markierung von Ironie. Diese beiden Aspekte sollen zunächst behandelt werden. Anschließend soll jene Eigenschaft als Bezugsgröße gewählt werden, die als Charakteristikum der traditionellen Interpunktion gilt: die Lesesteuerung bzw. Textgliederung. Zur Ergänzung werden Diskussionen referiert, die sich in Internet-Foren finden. Schließlich soll das Verhältnis von Emoticons und Interpunktion auf der Grundlage dieser Befunde zusammenfassend bewertet werden.

\section{Emotionalität}

\subsection{Emotionalität in der traditionellen Interpunktion}

Der Ausdruck von Emotionalität ist sicher nicht die herausragende Funktion der traditionellen Interpunktion. Dennoch gibt es mehrere herkömmliche Interpunktionszeichen, die zumindest eine Affinität zur Emotionalität aufweisen. Wir wollen hier drei dieser Zeichen behandeln:

Einen besonders auffälligen Bezug zur Emotionalität hat das Ausrufezeichen. Bereits die Bezeichnung „Ausrufezeichen“ konserviert die traditionelle Affinität dieses Graphems zum Exklamativsatz. Diese Beziehung weist zurück bis auf die rhetorische Figur der exclamatio, welche von Quintilianus (1. Jahrhundert n. Chr.) als Ausdruck solcher Gefühlsregungen wie z.B. Zorn (irasci), Freude (gaudere) oder Entrüstung (indignari) beschrieben wurde (vgl. Quintilianus IX.II.26-29; deutsch: Quintilianus 2006: 278-281). Dies wurde - wie unzählige andere rhetorische Konzepte - in den europäischen Sprachkulturen der Neuzeit intensiv reflektiert. ${ }^{6}$ Und gerade auch die Interpunktionslehren der frühen Neuzeit waren stark von der antiken Rhetorik geprägt (vgl. Rinas 2012). Für das Deutsche beschreibt etwa schon Friedrich Riederer in seinem erstmals 1493 veröffentlichten deutschsprachigen Spiegel der waren Rethoric den Gebrauch des Ausrufezeichens, welches er als „exclamativus” bzw. ,admira-

\footnotetext{
${ }^{5}$ Im ELL2, Bd. 2, s. v. Computer-Supported Writing, findet sich eine Formulierung, die diese Interpretation nahe legt: „Emoticons (such as smiley faces) are used as punctuation marks to indicate irony and tone.”

${ }^{6}$ Aus literaturwissenschaftlicher Sicht wird diese Rezeption der antiken Rhetorik in dem klassischen Werk von Curtius (1993[1948]) beschrieben. Zur Rezeption innerhalb der Grammatikschreibung vgl. etwa Scaglione (1981).
}

ISSN 1615-3014 
tivus" bezeichnet: Dieses Zeichen werde gebraucht, "wenn einer seiner red verwundrende ein scharpffen vßdruck thůt" (zitiert nach Riederer 1517: Blatt XLIb). Damit ist bereits auf jenes emotional-intensivierende Moment verwiesen, welches den Gebrauch dieses Zeichens bis in die Gegenwart bestimmt. So formuliert Duden (2000: 30): "Das Ausrufezeichen verleiht dem Vorangehenden einen besonderen Nachdruck” (vgl. Amtliches Regelwerk 2006: §69).

Theoretisch bestünde die Möglichkeit, den Gebrauch des Ausrufezeichens stärker zu grammatikalisieren, indem man es nicht durchgehend mit dem Moment des Nachdrucks korreliert, sondern (auch) zur generellen Markierung der grammatisch-pragmatisch definierten Einheit des Aufforderungssatzes gebraucht. In den maßgeblichen Interpunktionslehren wurde dies aber nie konsequent durchgeführt. So wird zwar in älteren Duden-Auflagen durchaus konstatiert, dass das Ausrufezeichen nach Aufforderungssätzen stehe, doch wird dies gleich dahingehend eingeschränkt, dass bei Aufforderungssätzen ohne Nachdruck der Punkt stehen könne, ${ }^{7}$ wie etwa die folgenden Beispiele nach Duden (1986: 24) illustrieren:

Verlassen Sie sofort den Raum, wenn Sie sich nicht anständig benehmen können! VS.

(2) Servieren Sie jetzt bitte den Nachtisch.

Somit bleibt der Nachdruck das dominierende Moment. Auch Konrad Duden (1876: 7) äußerte sich in seinem ersten Entwurf einer Interpunktionslehre in diesem Sinne: „Das Ausrufezeichen steht am Schlusse solcher Sätze, die in der lebendigen Rede mit dem Ausdruck lebhafterer Gemütsbewegung gesprochen werden. Auf die Form [Hervorhebung im Original] der Sätze, ob sie nämlich Aussagesätze, Wunschsätze, Heischesätze oder Fragesätze sind, kommt es dabei nicht an."

Als weiteres Interpunktionszeichen mit einem Bezug zur Emotionalität sei hier der Gedankenstrich genannt. Dieser wurde erst im 18. Jahrhundert in die Interpunktionstheorie eingeführt (vgl. Höchli 1981: 299f.; Baudusch 1986: 55). Heynatz (1782: 59, 1803: 94f.) bezeichnet ihn als „Pause oder Gedankenstrich“ und definiert ihn als "Zeichen einer abgebrochenen Rede" (1803: 94). Diese Definition rückt den Gedankenstrich in eine direkte Beziehung zur traditionellen rhetorischen Figur der Aposiopese (vgl. Drews 1992), des „Gedankenabbruchs“, was Heynatz auch ausdrücklich hervorhebt. Und diese Figur wird bereits bei Quintilianus IX,2,54f. mit Emotionalität korreliert: "Die Aposiopese [...] stellt schon als solche eine Gefühlsbewegung [adfectus] zur Schau, sei es Zorn [ira], so: 'Euch will ich! - erst aber gilt's, das Toben der Fluten zu schlichten', sei es aus Sorge [...], sei es auch, um zu etwas anderem überzugehen" (nach der lateinisch-deutschen Ausgabe von Rahn (= Quintilianus 2006: 292f.)).

Auch im Duden (1986: 30) wird der Gebrauch des Gedankenstrichs beim "Abbruch der Rede" behandelt; der Duden (2000: 40) spricht vom "Verschweigen eines Gedankenabschlusses". ${ }^{8}$

\footnotetext{
${ }^{7}$ Vgl. auch Amtliches Regelwerk (2006: §67, E2). Diesbezüglich hat die Rechtschreibreform von 1996 also zu keiner Änderung geführt. Die älteren Duden-Auflagen werden hier nur deshalb zitiert, weil sie in ihren Ausführungen teils noch expliziter sind. Dies gilt auch für die weiter unten diskutierten Beispiele. Generell werden hier in diesem Beitrag elementare Interpunktions-Phänomene behandelt, die von der Rechtschreibreform nicht betroffen sind.

${ }^{8}$ Das Amtliche Regelwerk (2006) berücksichtigt diese Verwendung allerdings nicht.
} 
Die Figur der Aposiopese kann jedoch in der deutschen Schriftsprache nicht nur durch den Gedankenstrich, sondern alternativ auch durch die Auslassungspunkte markiert werden:

(3) "Sei still, du-!", schrie er ihn wütend an.

(4) "Sei still, du ...!", schrie er ihn wütend an. (vgl. Duden 2000: 29, 40)

Auch diese wurden erst spät, wohl erst zu Beginn des 19. Jahrhunderts, in die Orthographien eingeführt (vgl. Höchli 1981: 300f.; Baudusch 1986: 41). Die Affinität zur Emotionalität ist auch hier offenkundig.

\subsection{Wie emotional sind Smileys?}

Die Feststellung, dass Smileys einen Bezug zur Emotionalität aufweisen, bedarf scheinbar keiner Begründung, denn wie bereits in der Einleitung gezeigt wurde, sind sie ja gerade durch diesen Bezug funktional bestimmt, ${ }^{9}$ was auch in dem Terminus Emoticon deutlich hervorgehoben wird. Dass die Dinge dennoch nicht ganz so einfach liegen, wird deutlich, wenn man sich die Frage stellt, welchen Status solche Darstellungen wie The Unofficial Smiley Dictiona$r y$ besitzen: Sind solche Darstellungen vom Anspruch her normativ oder deskriptiv? Mit anderen Worten: Werden hier - tendenziell normative - Empfehlungen für den SmileyGebrauch formuliert, oder wird das Ziel verfolgt, die wichtigsten/gängigsten Gebrauchsweisen im Internet korrekt zu beschreiben? ${ }^{10}$ Falls hiermit ein deskriptiver Anspruch vertreten wird, so muss dem entgegenhalten werden, dass ein ausschließlicher Rekurs auf Emotionen inadäquat, dass mithin etwa eine Charakterisierung des Ur-Smileys als „ein Zeichen für gute Laune" empirisch unzulänglich ist. Dies ist in der Literatur bereits mehrfach hervorgehoben worden, ${ }^{11}$ besonders nachdrücklich von Runkehl, Schlobinski und Siever (1998: 97-99). Diese Autoren unterscheiden drei Funktionen der Smileys, die hier anhand konstruierter Beispiele illustriert werden sollen:

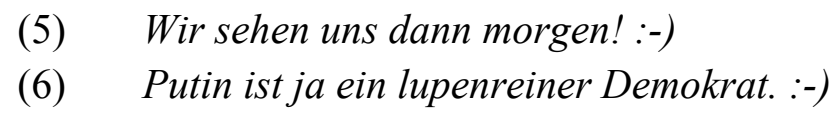

Beim expressiv-emotiven Gebrauch geht es um den direkten Ausdruck von Gefühlen: 'Ich freue mich darauf, dass wir uns morgen sehen.'

Beim evaluativen Gebrauch kommentiert und bewertet der Schreiber mit dem Smiley seine eigene Aussage: 'Die Feststellung, dass Putin ein lupenreiner Demokrat ist, ist nicht ernst gemeint.'

Beim kommunikativ-regulativen Gebrauch geht es um „eine spezifische Art von Adressatenhonorifikation, durch die der Kommunikationsmodus als positiv-freundschaftlich markiert wird” (Runkehl/Schlobinski/Siever 1998: 98).

\footnotetext{
${ }^{9}$ In diesem schlichten Sinne wird etwa in Ferretti (2004: 251) ein Emoticon definiert als „Symbol zur Übermittlung von Emotionen, z.B.: :-) für 'Spass'”.

${ }^{10}$ Dieses Changieren zwischen normativer und deskriptiver Darstellung ist natürlich ein Grundproblem jeglicher Sprachbetrachtung und keineswegs ein spezifisches Problem des Smiley-Gebrauchs. In Bezug auf Schreibkonventionen wird in diesem Sinne mitunter auch differenziert zwischen der (normativen) Orthographie und der (deskriptiven) Graphematik; vgl. etwa Dürscheid (2012: 126f.).

${ }^{11}$ So warnt Crystal (2006: 37, Fn. 15) vor einer direkten Korrelation von Smileys und den mit ihnen ikonisch abgebildeten Emotionsäußerungen.
} 
Ob diese Auflistung der Funktionen bereits erschöpfend und hinreichend differenziert ist, muss hier im Moment nicht diskutiert werden. Einstweilen genügt es, zu konstatieren, dass Smileys offenkundig keineswegs ausschließlich zum Ausdruck von Emotionen gebraucht werden, sondern dass sich weitere (,abstraktere“) Gebrauchsweisen herausgebildet haben. Ähnliches wurde auch in der Geschichte der Interpunktion beobachtet. So lassen sich etwa verschiedene Entwicklungsstufen und Abstraktionsgrade beim Gebrauch der konventionellen (nicht-modalisierenden) Anführungszeichen unterscheiden (vgl. Bredel 2011: 57-59):12

- Verweis auf zitierte Passagen:

$$
\text { „, Gott ist tot”, sagt Nietzsche. }
$$

- Verweis auf Werktitel:

(9) „Die Räuber” wurden 1782 uraufgeführt.

(Hier kommt es zur Referenzverschiebung: „Die Räuber” verweist nicht auf eine Gruppe von Menschen, sondern auf ein Drama.)

- Auszeichnung von Objektsprache im Rahmen einer metasprachlichen Ausführung:

$$
\text { „Blume” hat fünf Buchstaben. }
$$

In einem immer abstrakteren Maße wird hierbei auf das Medium Schrift rekurriert (zitierte schriftliche Aussage - schriftliches Werk - schriftliche Realisierung): „Die Geschichte der konventionellen Anführungszeichen [...] erweist sich als zunehmendes Eindringen der Schrift in das semiotische System der Sprache" (Bredel 2011: 59).

Es scheint somit, dass Smileys und Interpunktionszeichen im Schreibgebrauch zumindest teilweise ganz ähnlichen Entwicklungstendenzen folgen.

\section{$3 \quad$ Ironie}

\subsection{Ironie beim Interpunktionsgebrauch}

Auch die Markierung von Ironie ist sicher keine zentrale Aufgabe der Interpunktion. Dennoch gibt es - mindestens - zwei Satzzeichen, die diese Funktion ausüben können:

Die schon in Abschnitt 2.1 angesprochenen Auslassungspunkte können auch in einer „Andeutungsfunktion“" gebraucht werden (vgl. Bredel 2011: 46f.), womit auch markiert werden kann, dass die vorangegangene Äußerung nicht allzu ernst und wörtlich zu nehmen ist. Intensiv wurde diese Möglichkeit beispielsweise von dem Sachbuchautor Walter R. Fuchs genutzt. Hier nur zwei - recht willkürlich ausgewählte - Beispiele:

(11) „Die zumeist etwas nüchterner denkenden Techniker vermissen bei diesen brillanten hochabstrakten Theorien der Mathematiker allerdings eine gewisse 'Anwendungsfreundlichkeit'..." (Fuchs 1968: 17).

(12) „Es ist zwar leicht einzusehen, daß es weit mehr Geschick erfordert, eine Etüde von Fréderic Chopin gedankenlos am Klavier herunterzuklimpern als ein schlichtes Kinderliedchen wie 'Fuchs, du hast die Gans gestohlen'. Musiklehrer werden jedoch bestätigen, daß auch so etwas nicht ausgeschlossen ist...” (Fuchs 1968: 47).

\footnotetext{
12 Auch die Anführungszeichen wurden erst im 18. Jahrhundert in die Interpunktionslehre eingeführt; vgl. Höchli (1981: 301f.) und Baudusch (1986: 172).
} 
Schon in Abschnitt 2.2 wurde der konventionelle Gebrauch der Anführungszeichen angesprochen. Daneben gibt es auch einen modalisierenden Gebrauch, mit dem der Sprecher eine gewisse Distanzierung von der von ihm gewählten Formulierung zum Ausdruck bringt (vgl. Bredel 2011: 60f.). Für diesen Kontext konstatiert Duden (2000: 25) explizit eine Verwendung als ,,ironische Hervorhebungen":

(13) Dieser ,treue Freund” verriet ihn als erster. (Vgl. auch Amtliches Regelwerk 2006: $\S 94,4)$

\subsection{Ironie beim Smiley-Gebrauch}

Die Korrelierung von Smileys mit Ironie scheint ebenfalls keines Beweises zu bedürfen, denn auch diesbezüglich sind sich alle Darstellungen zum Smiley-Gebrauch einig (vgl. die Einleitung). Dies geht auch aus der im Abschnitt 2.2 referierten Analyse von Runkehl, Schlobinski und Siever (1998: 97-99) hervor, denn der dort beschriebene evaluative Gebrauch erfasst ja vorwiegend einen Verweis auf „nicht allzu ernst zu nehmende“, also eben ironisch gemeinte Aussagen.

Man könnte hierin eine vom expressiv-emotiven Gebrauch abgeleitete abstraktere Verwendungsweise sehen. Rein sachlogisch dürfte das auch stimmen. Historisch gesehen scheint der ironische Gebrauch allerdings von Anfang an mit den Smileys assoziiert gewesen zu sein, ja er dürfte ihre Einführung sogar wesentlich motiviert haben. ${ }^{13}$

Beim ironischen Gebrauch der Smileys sind distributionelle Gemeinsamkeiten mit den in 3.1 beschriebenen traditionellen Interpunktionszeichen unübersehbar:

i) Dem Auslassungszeichen in Andeutungsfunktion entspricht der satzfinal gebrauchte Smiley. Möglich wäre etwa ein Gebrauch wie:

Putin ist ja ein lupenreiner Demokrat. :-)

Putin ist ja ein lupenreiner Demokrat...

ii) Auch ein satzinterner ironischer Gebrauch von Smileys lässt sich belegen:

„Aber mit Klinsmanns frischen Wind - mussten auch neue Trikots her würg” [zitiert nach der Diskussionsseite Tradition oder rote Trikots?] ${ }^{14}$

Dieser entspricht funktional dem ironisch-modalisierenden Anführungszeichen:

Aber mit Klinsmanns "frischem Wind" mussten auch neue Trikots her würg

\section{$4 \quad$ Lesesteuerung}

\subsection{Interpunktion und Lesesteuerung / Relevante Einheiten}

In den Abschnitten 3 und 4 wurden solche Eigenschaften betrachtet, die als typisch für Smileys gelten. In diesem Abschnitt sollen umgekehrt die zentralen Charakteristika der her-

\footnotetext{
${ }^{13}$ So hat schon der angebliche Erfinder des Emoticons :-) Scott Fahlman diese Zeichenkombination 1982 als ,joke marker“ eingeführt (vgl. Orig-Smiley sowie Lischka 2007).

${ }^{14}$ Der Rektionsfehler findet sich im Original. Auch bei den anderen in dieser Arbeit angeführten Web-Belegen wurden die Fehler unkorrigiert übernommen. Die URLs dieser sowie der im Folgenden zitierten Diskussionsforen finden sich im Literaturverzeichnis am Ende dieses Beitrags.
} 
kömmlichen Interpunktion als Vergleichsmaßstab gewählt werden. Was sind diese Eigenschaften?

Die traditionelle Interpunktionslehre ist aus der Rhetorik hervorgegangen und war von der Antike bis weit in die Neuzeit - im deutschen Sprachraum bis ins 18. Jahrhundert - stark der rhetorischen Periodenlehre verpflichtet (vgl. Rinas 2012). Die Periode wurde hierbei verstanden als semantisch und rhythmisch abgeschlossene und eigenständige Einheit, welche unterteilt wurde in die weniger eigenständigen Einheiten Colon und Comma. Diese Untergliederung muss hier nicht eingehender behandelt werden. ${ }^{15}$ Wichtig ist für unseren Zusammenhang vor allem, dass mit der Hervorhebung der rhythmischen Eigenständigkeit das Moment der lautlichen Realisierung eine besondere Bedeutung erfährt. Dies kann bei einer genuin rhetorischen Konzeption auch kaum überraschen. Aber auch bei der Übernahme in die Grammatiken wurde dieses Moment beibehalten. Beispielsweise hebt Aelius Donatus in seiner Ars maior (4. Jh. n. Chr.) hervor, dass die Interpunktion für den Einsatz beim lauten Vorlesen (,,in lectione”) zu beachten sei (vgl. Donatus-Schönberger 2009: 38f.).

In neueren Interpunktionstheorien wird die Funktion der Interpunktion anders bestimmt. Nach Bredel (2011: 7) werden Interpunktionszeichen ,nicht mit Lautgesten verknüpft, sondern vom Leser in irgendeiner anderen Weise, verrechnet'. Sie verweisen nicht auf Entitäten außerhalb der Schrift, sondern dienen der Strukturierung schriftlicher Einheiten selbst bzw. ihrer Verarbeitung". Insbesondere der Verarbeitungsprozess wird von Bredel hervorgehoben, indem sie postuliert, dass die Interpunktion vor allem den Leseprozess stützt bzw. stützen sollte (ebd.: 23). In Bezug auf diesen Prozess unterscheidet Bredel (ebd.: 24f.) zwei Verfahren bzw. Aktivitäten, nämlich

- das Scannen als grobes Orientieren im Text, also etwa das spontane Erfassen von Einzelwörtern oder Absätzen;

- das Processing, d. h. das Zusammenfügen von Einheiten (etwa von Buchstaben zu Wörtern oder von Wörtern zu Wortgruppen)

Und diesen beiden Verfahren versucht Bredel (ebd.) die einzelnen Interpunktionszeichen zuzuweisen.

Auf den ersten Blick erscheint Bredels Charakterisierung der Interpunktion ganz anders geartet als der traditionell-rhetorische Ansatz. Bei genauerem Hinsehen erweisen sich die Unterschiede jedoch als gar nicht so groß. Wenn die traditionelle Rhetorik/Grammatik postuliert, dass die Interpunktion das laute Lesen, also das richtige Akzentuieren und Rhythmisieren, fördern soll, dann ist hiermit natürlich auch vorausgesetzt, dass die Interpunktion die Orientierung im Text erleichtern und damit dessen Interpretation optimieren soll, denn ohne diese Voraussetzung wäre eine adäquate lautliche Umsetzung ja gar nicht möglich. Bredels Charakterisierung ist insofern eher eine Präzisierung von konzeptionellen Grundlagen, die von Anfang an in der Interpunktionslehre angelegt waren.

Eine weitere Parallele zwischen traditionell-rhetorischen und neueren Interpunktionslehren besteht darin, dass beide die Interpunktion an sprachliche Einheiten koppeln. Dies ist im

\footnotetext{
15 Abrisse dieser Lehre bieten etwa Lausberg (1973: 458-467), Stolt (1990: 379-381), Crowley/Hawhee (2004: 286-289) und Rinas (2012: 20-23).
} 
Grunde trivial, denn es ist ja der eigentliche Sinn der Interpunktion, komplexe Texte in kleinere sprachliche Einheiten zu gliedern. Weniger trivial ist allerdings die Frage, welche Einheiten interpunktionsrelevant sind. Bredel rekurriert in ihrem auf Scannen und Parsing basierenden Ansatz auf Einheiten wie Wörter, Wortgruppen, Sätze und Absätze. Die traditionelle Lehre rekurriert vor allem auf die Einheiten Periode, Kolon und Komma. Dies wirft die Frage auf, in welcher Beziehung diese traditionellen Einheiten zu unseren heutigen stehen. Wir wollen diese Frage wenigstens kurz am Beispiel der Periode diskutieren.

Die traditionelle Auffassung der Periode als (rhythmisch/semantisch) abgeschlossene Einheit erinnert an die weit verbreitete Charakterisierung des Satzes als einer autonomen Einheit, so dass es nahe liegt, diese beiden Einheiten zu identifizieren (vgl. etwa Bünting/Bergenholtz 1989: 20-27). Diese Einschätzung wird auch durch die Geschichte der Interpunktionslehre gestützt, etwa dadurch, dass der Punkt bis ins ausgehende 18. Jahrhundert vorwiegend als „Periodenschlusszeichen“, ab dem 19. Jahrhundert dann zunehmend als „Satzschlusszeichen“ aufgefasst wurde (vgl. etwa Weise 1691: 246 oder Basedow 1756: 176 vs. Duden 1876: 6).

Mit dieser Identifizierung ist die Periode als Einheit aber noch keineswegs erschöpfend bestimmt. Einer anderen Interpretation zufolge ist die Periode ein erweiterter oder ausgeschmückter Satz (vgl. etwa Schmitthenner 1824: 27f.). Auch diese Sicht geht auf antike Ansätze zurück, denen zufolge die Periode betrachtet werden kann als eine Einheit, in der „mehrere Gedanken so aufeinander bezogen sind, dass anfangs eine Spannung entsteht, die am Ende ihre Auflösung erfährt" (Staab 2009: 1502). Gerade dies sollte mit dem Terminus Periode (,Umlauf') erfasst werden. Derartige Überlegungen finden sich etwa bei Quintilian (IX 4, 124) (=2006: 419).

Diese traditionelle Auffassung der Periode als eines komplexen, in sich abgeschlossenen gedanklichen Umlaufs findet aber noch in Definitionen einer weiteren (schrift-)sprachlichen Einheit ihren Widerhall, und zwar in den gängigen Definitionen des Absatzes. Dies illustriert etwa die folgende Absatzdefinition von Friedmann: ${ }^{16}$

Die Gesamtaussage, die der Absatz enthält, besteht aus einer Reihe miteinander verbundener Gedanken, von denen jeder durch einen selbständigen Satz ausgedrückt wird. Somit verfügt über eine gewisse semantische, inhaltliche Abgeschlossenheit nur der Absatz im ganzen. Einzelne Sätze als Absatzkomponenten bezeichnen nur Teile, Elemente dieser Gesamtaussage, darum sind sie semantisch nicht abgeschlossen, also synsemantisch.

(Friedmann 1972: 298)

Insofern kann die Periodenlehre auch als (partielle) Vorwegnahme der Absatztheorie angesehen werden, was bislang offenbar noch nicht reflektiert wurde (vgl. Rinas 2015).

Aus dem Gesagten folgt, dass das Konzept der Periode auf (mindestens) drei verschiedene Weisen mit heutigen sprachlichen Einheiten korreliert werden kann: mit Sätzen, mit Satzgefügen und mit Absätzen. Dies wird auch bei unseren weiteren Überlegungen zu berücksichtigen sein.

\subsection{Emoticons und Lesesteuerung? / Relevante Einheiten?}

Lässt sich die Funktion der Textgliederung bzw. Lesesteuerung auch für Smileys konstatieren? Das wäre dann der Fall, wenn auch mit Smileys „leserelevante“ sprachliche Einheiten

${ }^{16}$ Vgl. etwa auch die Darstellung der anglo-amerikanischen Absatztheorie bei Mautner (2011: 69-98). 
ausgegliedert werden. Dies führt zur Frage, an welchen Positionen im Text Smileys üblicherweise platziert werden. Hierzu gibt es in der Literatur nur wenige, zugleich jedoch auch widersprüchliche Aussagen.

Nach Crystal (2006: 39) werden Smileys ,placed after the final punctuation mark of a sentence". Der Gebrauch der Smileys wäre somit in gewisser Weise auf die Einheit des Satzes bezogen, wenngleich Smileys selbst einen Satz nicht abschließen. Es lassen sich allerdings durchaus Gegenbeispiele finden, wo Smileys offenbar selbst die satzabschließende Funktion übernehmen:

(19) „Das letztemal das ich so Augenschmerzen hatte war damals als Dortmund in lilaweiß gespielt hat -2 Traditionen im Fußball werden mittlerweile mit Füßen getreten, zudem denke ich, dass das rote Trikot auch zum Geldscheffeln dienen soll."

(Beispiele nach Tradition oder rote Trikots?)

Runkehl, Schlobinski und Siever (1998: 97) konstatieren für den speziellen Fall der ChatKommunikation: „Smileys stehen in der Regel turnfinal.” Hier ist also nicht der Satz, sondern der Turn die relevante Bezugseinheit. Dieser Argumentation liegen offenbar die folgenden Annahmen zugrunde:

i) Die Chat-Kommunikation weist zumindest partiell Gemeinsamkeiten mit der gesprochenen Sprache auf (vgl. auch Runkehl/Schlobinski/Siever 1998: 84, 99; Wilde 2002; HessLüttich/Wilde 2003: 169).

ii) Für die Analyse dieser dialogischen Textform ist der Turn eine geeignetere Kategorie als der Satz.

Beide Annahmen erfordern eine ausführlichere Diskussion, als sie hier gegeben werden kann. Auf die Diskussion von Annahme i) wollen wir ganz verzichten (vgl. hierzu etwa Storrer 2001; Dürscheid 2003, 2011). Annahme ii) soll zumindest kurz kommentiert werden.

Die These, dass der Turn und nicht der Satz eine zentrale Bezugseinheit der gesprochenen Sprache sei, wird im Rahmen der Gesprächsanalyse oft vertreten. Zur Stützung dieser These werden diverse Einsichten und Ergebnisse dieser Forschungsrichtung herangezogen. Dennoch ist diese Diskussion oft hermetisch, da die abgelehnte Satz-Konzeption von den TurnBefürwortern selten ernsthaft reflektiert wird (vgl. die Kritik von Hennig 2006: §II.1.1). Dies illustriert etwa ein Aufsatz von Fiehler (2003), in welchem nachdrücklich dafür plädiert wird, den Gesprächsbeitrag/Turn als die zentrale Einheit gesprochener Sprache anzusehen, wobei Fiehler diesen folgendermaßen definiert:

Ein Beitrag ist eine Äußerung, die mit Rederecht gemacht wird. Dass jemand einen Beitrag macht, heißt, dass er zu einem bestimmten Zeitpunkt mit Rederecht spricht. D. h. sein Recht zu reden, ist von den anderen Gesprächsteilnehmern ratifiziert bzw. wird nicht durch Handlungen irgendwelcher Art in Frage gestellt.

(Fiehler 2003: 149f.)

Fiehlers Argumentation zugunsten des Turn ist durchaus erwägenswert. Seine Ablehnung der Satz-Konzeption ist hingegen verwunderlich, da Fiehler sich mit dieser Konzeption gar nicht auseinandersetzt. Er macht sich nicht einmal die Mühe, ausführlicher darzulegen, was er unter 
einem Satz verstanden wissen möchte, sondern begnügt sich mit der beiläufigen Bemerkung, dass ein Satz im Rahmen seines Beitrags ,als Komplex aus Referenz und Prädikation” verstanden werden solle (ebd.: 149). Dies könnte vielleicht als - wenn auch oberflächliche Charakterisierung der Satztheorie des 19. Jahrhunderts durchgehen, wenngleich auch die ältere Tradition des Satzes wesentlich reichhaltiger war, wie etwa die Sammlungen von Ries (1931) und Seidel (1935) belegen. Fiehlers Satz-Charakterisierung ist jedoch gänzlich verzerrend, wenn es um neuere Satz-Definitionen geht, wie sie beispielweise von Müller (1985a, 1985b) und Hoffmann (1992) diskutiert werden.

Hätte Fiehler die Beiträge von Ries, Seidel, Hoffmann und Müller berücksichtigt, dann hätte sein Vergleich der Konzeptionen von Turn und Satz wesentlich differenzierter und ergiebiger ausfallen können. Außerdem hätte bei diesem Vergleich auch die in Abschnitt 4.1 angesprochene Einheit der Periode berücksichtigt werden können, die ja eine deutliche Affinität zur Satzkonzeption aufweist. Gerade aus Sicht der Gesprächsanalyse wäre eine Auseinandersetzung mit dieser ebenfalls gesprochen-sprachliche (intonatorische) Aspekte berücksichtigenden rhetorischen Einheit zweifellos lohnend. ${ }^{17}$

In Abschnitt 4.1 wurde auch kurz der Absatz (als eine mögliche Entsprechung des traditionellen Konzepts der Periode) behandelt. Auch im hiesigen Zusammenhang ist diese schriftsprachliche Einheit von Interesse: Wie stark ist die Affinität der Smileys zum Absatz? Unseres Wissens gibt es keine empirischen Untersuchungen zu dieser Frage, doch dürften auch diese lohnend sein. ${ }^{18}$

Zurück zur These von Runkehl, Schlobinski und Siever: Die Auffassung, dass Smileys turnfinal stehen, impliziert, dass sie nur am Ende eines Gesprächsbeitrags, also unmittelbar vor einem Sprecherwechsel auftreten können. Für die ohnedies auf kurze Beiträge beschränkte Chat-Kommunikation ist dies zumindest diskutabel, bei anderen schriftlichen Kommunikationsformen ist hingegen fragwürdig, ob das turn-Konzept überhaupt sinnvoll angewendet werden kann. Dies ist jedenfalls dann problematisch, wenn man den turn - wie etwa Fiehler (s. o.) - eng mit dem Rederecht in Verbindung bringt, da es kaum einleuchtend scheint, in analoger Weise ein „Schreibrecht“" zu postulieren.

Aber selbst in Bezug auf die Chat-Kommunikation ist die These von Runkehl, Schlobinski und Siever keineswegs vollkommen überzeugend, wie etwa schon das obige Chat-Beispiel (19) zeigt, wo das Smiley innerhalb eines Turns platziert ist und eine Satzgrenze markiert.

Die bisherigen Beispiele illustrieren, dass Smileys an Positionen auftreten können, wo traditionelle Satzschlusszeichen (Punkt, Fragezeichen, Ausrufezeichen) stehen, wobei sie diese Zeichen ergänzen, aber mitunter auch ersetzen können. Dies geschieht häufig an markierten Endpositionen, gerade auch am Absatzende, doch sind auch Verwendungen innerhalb von Absät-

\footnotetext{
${ }^{17}$ Dies scheint allgemein ein Desiderat zu sein. So reflektiert beispielsweise auch Hennig (2006: 22, 160f., 213) in ihrer grundlegenden Studie zur Grammatik der gesprochenen Sprache mitunter die rhetorische Tradition, doch bleibt die Kategorie der Periode unberücksichtigt, und zwar auch im Rahmen der Diskussion, ob Satz oder Turn die grundlegende Einheit der gesprochenen Sprache sei (ebd.: 147-160).

18 In diesem Sinne ist auch der Titel unseres Beitrags bewusst vieldeutig: Das einigermaßen „schillernde“ Konzept der Periode erlaubt mehrere Deutungen und lässt insofern offen, auf welche sprachlichen Einheiten die Emoticons rekurrieren. Zudem soll natürlich die Verbindung des älteren Perioden-Begriffs mit dem relativ neuen Phänomen der Emoticons andeuten, dass hier zwei unterschiedliche Traditionen kontrastiert werden.
} 
zen und sogar im Satzinnern möglich. Dies dürfte jedoch bei den Smileys im engeren Sinne, d. h. bei den grafischen Gesichtsdarstellungen, weitaus üblicher sein als bei den aus ASCIIZeichen zusammengesetzten Emoticons, da letztere aufgrund ihrer Komplexität den Lesefluss beeinträchtigen können. Darüber hinaus nutzen Emoticons weitgehend den gleichen Zeichenbestand wie die traditionelle Interpunktion, was zu Unklarheiten oder Verwechslungen führen kann, die beim Gebrauch von Smileys nicht auftreten. Besonders irritierend ist es, wenn Emoticons und Interpunktionszeichen in unmittelbaren Kontakt treten. Beispiele dieser Art finden sich teilweise sogar in der Fachliteratur, vgl. etwa:

„In den von uns untersuchten 131 Beispielartikeln (Newskorpus) finden sich 24

Vorkommen von :-), 18 Vorkommen von :) und 12 Vorkommen von ;)." (Haase et al. 1997: 64)

In diesem Passus ist das Standard-Smiley :-) unmittelbar mit einem Komma verbunden, das im Rahmen der üblichen orthographischen Norm als Koordinations-Mittel verwendet wird. Kombinationen dieser Art werden auch in Internet-Diskussionen kritisiert:

„Ein Punkt danach sieht einfach nicht gut aus: :-).”

(haben-smiley-eine-satzzeichen-funktion)

(22) „Ganz blöd ist das mit Klammern (so wie hier zum Beispiel ;-)).”

(haben-smiley-eine-satzzeichen-funktion)

Diese Belege illustrieren bereits, dass die Beziehung von Smileys und Interpunktion auch in Internet-Foren diskutiert wird. Dies soll im folgenden Abschnitt ausführlicher behandelt werden.

\section{$5 \quad$ User-Diskussionen im Internet}

In den letzten Jahren wurde das Verhältnis von Smileys/Emoticons und Interpunktionszeichen wiederholt in Internetforen diskutiert. Im Folgenden sollen einige der Standardargumente referiert und kommentiert werden. ${ }^{19}$ Gegenstand diese Kapitels sind also „laienlinguistische“20 Reflexionen über den Status und den Gebrauch von Emoticons.

Die Diskussionen im Internet kreisen im wesentlichen um drei miteinander zusammenhängende Fragenkomplexe:

i) Welcher sprachlichen Ebene lassen sich Smileys zuordnen? Sind sie Bestandteile eines Satzes?

Hier wird häufiger argumentiert, dass Smileys Emotionen ausdrückten, woraus ein Unterschied zu Interpunktionszeichen abgeleitet wird:

„Also ich seh Smileys als Gemütsuntermalung/nachträgliche Betonung des Vorrange gangenem. ;D [...] Da die Mimik und Gestik ja beim Schreiben völlig entfällt und spe zielle Formen der Erzählung, die man sonst nur durch eben solche körperlichen Gesten

19 Bei dieser Auswertung wurden Diskussionen auf deutschsprachigen Seiten berücksichtigt, und zwar: DasSmiley-Problem / Gibt es eine Smileyregelung in der Rechtschreibung? / haben-smiley-eine-satzzeichen-funktion / Smilies-und-Satzzeichen. Alle sprachlichen Fehler in diesen Diskussionsbeiträgen wurden unverändert übernommen.

${ }^{20}$ Zur „Laienlinguistik“"vgl. Antos (1996). 
zum Ausdruck hätte bringen können, dadurch zu kurz kommen, untermauert man das Ganze durch einen Smiley. [...] Also sind Smileys nicht als Satzzeichen zu verstehen, sondern als SATZVERSTÄRKER.” (haben-smiley-eine-satzzeichen-funktion)

„Ein Smilie ist normalerweise eine Zusatzinformation wie der davor stehende Text zu interpretieren ist, kann aber unter umständen auch eine eigenständige Aussage sein. Auf jeden Fall ist er nicht Teil des Satzes und gehört deshalb hinter einen Punkt." (Smilies-und-Satzzeichen)

(25) „Ich würde den Smiley immer ausserhalb des Satztes anbringen. Da er ja nicht als sprachliche Äußerung zu dem Satz gehört sondern den Satz mit einer entsprechenden Emotion bereichern soll. ;)" (Gibt es eine Smileyregelung in der Rechtschreibung?)21

Diese Argumentation ignoriert jedoch, dass die Funktion des Smileys sich nicht auf das Signalisieren von Emotionalität beschränkt (vgl. Abschnitt 2.2). Außerdem wird hierbei nicht beachtet, dass auch die klassischen Interpunktionszeichen mit Emotionalität assoziiert sind (vgl. Abschnitt 2.1).

ii) Haben Smileys funktionale Gemeinsamkeiten mit Interpunktionszeichen? Können Smileys $\underline{\text { Satzzeichen ersetzen? }}$

Einige User lehnen den Gebrauch von Smileys anstelle von Interpunktionszeichen ab. Auch dies basiert zumeist auf der Voraussetzung, dass den Smileys ein anderer Status als den Interpunktionszeichen zukomme:

(26) „Für mich ist das eine Schrift- und das andere Bildsprache, ergo können sie keine Satzzeichen ersetzen, oder?" (Das-Smiley-Problem)

(27) „Findet ihr nicht auch, dass ein fragender Smilie ein Fragezeichen mehr als ausreichend ersetzt??? [...]"

[Antwort eines anderen Users:] „noe, das Geschriebene gibt quasi das wieder, was man in der entsprechenden Situation von Angesicht zu Angesicht zu jemandem sagen wuerde. Das Fragezeichen (Punkt, etc.) entspricht dann der jeweiligen Betonung am Satzende. Der Smiley steht in dem Fall aber fuer den fragenden Gesichtsausdruck. [...] Das Fragezeichen ersetzt also die akustische Information, waehrend der graphische Smiley die virtuelle Transformation des visuellen Eindrucks darstellt." (Das-SmileyProblem)

Erneut wird also eine enge Korrelierung der Smileys mit dem ikonisch-bildhaften Ausdruck von Emotionen vorausgesetzt. Dies ist aber ein Postulat, das die abstrakteren Verwendungen der Smileys nicht berücksichtigt (s. o.).

Andere User halten eine „Verdrängung“ von Satzzeichen durch Smileys für zulässig, wobei zumeist ästhetische Argumente angeführt werden:

(28) „Ein Punkt danach sieht einfach nicht gut aus: :-). Also: Obwohl es vermutlich falsch ist, lasse ich das Satzzeichen weg." (haben-smiley-eine-satzzeichen-funktion)

(29) „Ich finde ein . und ein folgendes Smilie sieht doof aus. Ein Smilie und danach ein Punkt ist in meinen Augen ein Zeichen dafür, dass jemand wohl nen, zumindest teilweise, miesen Geschmack hat" (Smilies-und-Satzzeichen) 21 Auch in der linguistischen Fachliteratur wird häufiger hervorgehoben, dass Smileys eher als zusätzlicher
Kommentar zu deuten seien (vgl. etwa Dürscheid 2006: 107 und Wirth 2006: 123). 
Diese Einschätzungen sind natürlich subjektiv.

iii) Welche graphotaktischen Bedingungen gelten für den Smiley-Gebrauch?

Bei diesem Problem lassen sich mehrere Teilfragen unterscheiden.

iii-a) Sollten Smileys innerhalb oder außerhalb von Sätzen platziert werden? Sollten sie generell vor oder hinter Interpunktionszeichen stehen?

Diese Frage wird meistens auf die Abfolge von Satzpunkt und Smiley beschränkt. Häufiger wird die Positionierung des Smileys nach dem Punkt abgelehnt, weil der Punkt als Abschlusssignal interpretiert wird:

(31) „ [...] das [i. e. ein Smiley nach einem Satzpunkt, K.R./V.U.] wirkt so, also ob man den Satz zu Ende sprechen würde und dann nachträglich eine Grimasse ziehen würde. Unnatürlich und aufgesetzt."

(haben-smiley-eine-satzzeichen-funktion)

(32) „Der Punkt bildet eine Zäsur, einen Einschnitt. Sonst könnte man ja auch z.B. mit einem Semikolon weitermachen. Wenn aber nach diesem kurzen Innehalten ,plötzlich" ein Smiley folgt - dann wirkt das in der Tat irgendwie seltsam, wie eine kleine Fratze. Würde ich deswegen auch nie so machen." (haben-smiley-eine-satzzeichen-funktion)

Ein zusätzliches Argument gegen diese Abfolge ist, dass sie nicht deutlich erkennen lasse, worauf sich das Smiley beziehe:

(33) „[...] das [i. e. wenn man das Smiley hinter den Punkt setzt, K.R./V.U.] könnte man aber auf die Idee kommen, der Smilie gehört schon zum nächsten Satz - zumindest wenn er in der gleichen Zeile wie der Smilie beginnt." (Gibt es eine Smileyregelung in der Rechtschreibung?)

(34) „find das nur manchmal bissel schwierig wenn der smiley orgendwo inmitten steht, dann weiß ich immer nicht zu welchem satz der nun gehört,... denn je nach smileyx kann nen satz unterschieldiche bedeutungen haben. ;)"

(Gibt es eine Smileyregelung in der Rechtschreibung?)

Die Frage des Bezugsbereichs reflektiert auch die folgende Argumentation:

„Warum sollte er ausserhalb stehen, ein Smilie ist doch auch nur eine Piktogramm bestehend aus Satzzeichen, welches eine Emotion o.ä., visuell ausdrücken soll - ähnlich einer Anmerkung, die man in Klammern setzt ala „, (lacht)“. sowas schreibt man auch nicht ausserhalb. Ist der Bezug der ausgedrückten Emotion innerhalb des Satzes zu finden, sollte man es auch innerhalb des Satzes schreiben, der Punkt also als Abschluss des Satzes dahinter setzen, damit ein einheitliches, zusammengehöriges Gefüge entsteht.

Weiss ehrlich nicht, wie die Erklärung dafür lauten sollte, es nach dem Endsatzzeichen zu schreiben, zumal Smilies innerhalb eines Satzes als emotionale Randnotiz auch nicht so selten sind (zumeist am Ende eines Nebensatzes), da setzt man doch auch das Komma dahinter und nicht davor?" (Gibt es eine Smileyregelung in der Rechtschreibung?) 
Hier wird also auch die Tatsache reflektiert, dass Smileys satzintern stehen können (vgl. Abschnitt 3.2).

Argumente für die Positionierung des Smileys hinter dem Punkt finden sich selten; sie sind eher ästhetischer Art:

(36) „Ich persoenlich, taete den punkt vorm smiley machen. ;) Find ich persoenlich schoener."(Gibt es eine Smileyregelung in der Rechtschreibung?)

", [Wenn man zusätzlich zum Smiley einen Punkt setzt], dann wohl hinter den Smiley, aber dort sieht es dann so veroren aus." (haben-smiley-eine-satzzeichen-funktion)

iii-b) Sollten sie direkt mit Interpunktionszeichen verbunden oder durch einen Leerschritt von ihnen getrennt werden?

Diese Frage wird nur in einer der untersuchten Diskussionen explizit angesprochen:

(38) „Zweite Frage ist, ob sich der Smilie unmittelbar nach dem Satzzeichen anschließen muss oder ob ein Leerzeichen dazwischen sein soll.” (Das-Smiley-Problem)

Interessanterweise wird dieses Problem verknüpft mit der Frage nach dem Bezugsbereich der Smileys, wie im folgenden Abschnitt gezeigt wird.

iii-c) Wie kann der Bezugsbereich von Smileys graphotaktisch am besten markiert werden?

In den hier untersuchten Diskussionen findet sich zu diesem Problem nur ein Vorschlag:

(39) „Ich bin aber der Meinung, der Smilie gehört unmittelbar hinter den zugeordneten Abschnitt, um die Trennung zum nachhergehenden Satz anzuzeigen. Schließlich bezieht sich der Smilie regelmäßig auf das vorhergehende und sollte daher nicht mittig stehen. Ich würde die Fußnotenregeln analog anwenden: Bezieht er sich auf ein Wort, steht er unmittelbar dahinter. Bezieht er sich auf einen Satz oder einen Satzteil, steht er ohne Leerzeichen hinter dem Satzzeichen. Ein Leerzeichen ist zu machen, wenn er sich auf den ganzen Absatz beziehen soll." (Das-Smiley-Problem)

Dies wird durch das folgende Beispiel erläutert:

(40) „Zicke geht mir auf den Sack. Balu hingegen macht intelligente Freds auf, drum mag ich ihn.” (Das-Smiley-Problem)

Damit wären die Einwände gegen die Platzierung des Smileys nach dem Satzpunkt weitgehend hinfällig.

Über diesen Vorschlag hinausgehend, ließe sich auf analoge Weise auch der Bezugsbereich eines Smileys markieren, wenn dieses sich auf eine Wortgruppe innerhalb eines Satzes bezieht. Diesbezüglich gibt es nämlich weiterhin Unklarheiten, wie etwa das folgende Beispiel zeigt:

(41) Seine neue Erfindung ist hier das Tagesgespräch.

Bezieht sich der Ironie-Smiley hier auf Erfindung oder auf neue Erfindung? Mit den paarigen Anführungszeichen ist der Bezug deutlicher:

(42) Seine neue 'Erfindung' ist hier das Tagesgespräch.

(43) Seine 'neue Erfindung' ist hier das Tagesgespräch.

Man könnte somit erwägen, auch paarige Smileys einzuführen: 


$$
\begin{aligned}
& \text { Seine neue } @ \text { Erfindung } @ \text { ist hier das Tagesgespräch. } \\
& \text { Seine - Nneue Erfindung-ist hier das Tagesgespräch. }
\end{aligned}
$$

In den hier untersuchten Internet-Diskussionen wurden solche Vorschläge allerdings nicht unterbreitet.

\section{6 Überlegungen zum Status der Smileys}

In diesem abschließenden Abschnitt sollen unter Einbeziehung des bisher Erarbeiteten einige Überlegungen zum schriftlinguistischen Status der Smileys angestellt werden.

Wie in Abschnitt 5 demonstriert wurde, wird auch unter Internet-Usern die Frage diskutiert, ob Smileys Gemeinsamkeiten mit den traditionellen Interpunktionszeichen aufweisen. Diese Frage ist berechtigt. Was die Markierung von Emotionalität und Ironie angeht, lassen sich durchaus funktionale Überschneidungen zwischen Smileys und Interpunktionszeichen konstatieren (vgl. die Abschnitte 2 und 3). Ob Smileys ebenso wie Interpunktionszeichen auch zur Lesesteuerung beitragen, ist weniger klar, doch scheinen auch Smileys eine gewisse Affinität zur Einheit des Satzes - und Absatzes - zu haben (vgl. Abschnitt 4). Aus diesen Befunden kann gewiss nicht abgeleitet werden, dass Smileys eine ernsthafte Konkurrenz zum traditionellen Interpunktionssystem darstellen, aber es wäre zumindest diskutabel, sie als eine gewisse Ergänzung des traditionellen Systems anzusehen. Dies wirft allerdings die Frage auf, ob eine solche Ergänzung überhaupt sinnvoll ist. Diese Frage kann letztlich nur im Rekurs auf sprachlich-kommunikative Leitvorstellungen vorgenommen werden, also in Bezug auf eine bestimmte Norm. Gerade aus dieser Sicht wird die Leistung der Smileys in der Fachliteratur häufiger kritisch beurteilt. Nach Wirth (2005: 82) hat die Einführung von Smileys ,eine Inflation expliziter Selbstkommentare” zur Folge. Hess-Lüttich und Wilde (2003: 169) interpretieren den Smiley-Gebrauch als Ausdruck von Konfusion und Unsicherheit: „Wenn der Chatter seine Sätze selbst nicht mehr versteht, paraphrasiert er geschwind, Verstehen heischend mit vorgestelltem ,ich meine“ oder angehängtem Smiley, das um Nachsicht bittet, aber meist schroff mit Kaskaden von Fragezeichen beantwortet wird." Und Crystal (2006: 39) wirft die Frage auf, ob Smileys nicht nutzlos (,futile”) seien.

Diese Einschätzungen erinnern an die zuweilen von Sprachpflegern vorgetragene Pauschalkritik an Partikeln als „Flickwörtern“,22 eine Einschätzung, die von der Partikelforschung zurückgewiesen wurde mit dem Hinweis darauf, dass Partikeln sehr wohl wichtige kommunikative Formen erfüllen können. Dies schließt freilich die Möglichkeit missbräuchlicher Verwendungen sowie den hypertrophischen Gebrauch keineswegs aus. Ebenso gibt es beim Smiley-Gebrauch sicher Unsicherheiten und Übertreibungen (vgl. Crystal 2006: 39f.), die aber nicht dazu berechtigen, ihre Verwendung pauschal zu verurteilen.

Manche Unsicherheiten beim Smiley-Gebrauch könnten auch darauf zurückgeführt werden, dass es keine verbindlichen und klar formulierten Verwendungs-Regeln gibt. Ähnliches lässt sich über die ältere deutsche Interpunktion sagen. Insbesondere der Komma-Gebrauch galt bereits im 18. Jahrhundert als besonders schwierig, und er war zugleich oft unpräzise beschrieben. So kennzeichnet das Komma (der „Beystrich”) laut Gottsched (1776: 37) „die

\footnotetext{
22 Tatsächlich sind aber auch die Einschätzungen vieler Sprachpfleger längst nicht so einseitig abwertend, wie dies von Linguisten oft unterstellt wird (vgl. hierzu Rinas 2011: 99-104).
} 
kleinsten Trennungen gewisser Wörter, die nicht zusammen gehören”. Diese reichlich vage Regel ergänzt Gottsched (ebd.) durch folgende nicht minder vage Anweisung: 23 „Man muß sich nur in Acht nehmen, daß man der Sache weder zu viel, noch zu wenig thue; sondern die Mittelstraße halte. Zuviel Beystriche hemmen das Lesen zu sehr; zu wenige aber machen eine Schrift nicht deutlich genug; weil man nicht erinnert wird, wo man ein wenig still halten soll."

Angesichts solcher Regeln verwundert es nicht, dass so mancher Schreiber im 18. Jahrhundert Probleme hatte, beim Komma-Gebrauch das „rechte Maß“ zu finden. Einen solchen Fall schildert Heynatz (1782: 53): „Der allersonderbarste Interpunktator, den ich je gefunden habe, ist der nicht unbekannte Hempel, der Herausgeber der Gundlingischen vollständigen Historie der Gelahrtheit [...] Dieser macht oft fast hinter jedem Worte ein Komma." Zum Beleg zitiert Heynatz aus Hempels (nicht-paginierter) Vorrede in Gundling (1734): ${ }^{24}$

„Ob ich nun zwar, solcher Gestalt, Nichts ermangeln lassen, Was, zur Vollständigkeit des Wercks, contribuiren kan ; Auch, zu dem Ende, bereits bey diesem Tomo , einige mit untergeschlichene Druck-Fehler und andere Errata, die mir, beym Durchlesen, vorgekommen sind, angemercket habe ; Als womit, künftig, auch Ratione dieses Erstern Tomi, soll continuiret werden ; So wolle mir dennoch Niemand imputiren, daß ich, nicht so, verwegen bin, und das Werck etwa, ganz ohne Mangel und Irrthümer, ausgebe."

(Hempel (1734) zit. nach Heynatz (1782: 53))

Ähnlich wie Hempel den Komma-Gebrauch übertrieb, so pflegen heute viele Internet-User Smileys inflationär zu verwenden. Insofern kann es nicht verwundern, dass es auch im Internet Bemühungen gibt, den Smiley-Gebrauch auf das „,rechte Maß“ zu reduzieren. Allerdings sind diese Anweisungen oft auch nicht präziser sind als Gottscheds Ausführungen zum Komma-Gebrauch. Solche Regeln finden sich vor allem in Anleitungen zur höflichen und angemessenen Internet-Kommunikation, den sogenannten „Netiquettes“. So gehört nach Sjurts (2006: 152) zur Netiquette gewöhnlich ein ,sparsamer Umgang mit Emoticons” (vgl. etwa auch Alexander 2006: 143f.; Netiquette: Smileys vermeiden und die Wortwahl abwägen; NETiquette - deine Etikette im Netz). Einen Grund für diese Forderung nennt Sjurts nicht. Man könnte sie aber mit dem von Runkehl, Schlobinski und Siever (1998: 56) für die Netiquette referierten Grundsatz in Verbindung bringen, beim Umgang mit Ironie vorsichtig zu sein: Ständiger Einsatz von Ironie führt dazu, dass man nicht mehr ernst genommen wird. Ein unablässiger ironisierender Smiley-Gebrauch könnte ebendies zur Folge haben. In diesem Sinne konstatiert auch Netiquette beim E-Mailing, dass Smileys „bei zu häufigem Gebrauch stark an Wirkung" verlören. Derartige wirkungsorientierte Mahnungen erinnern wiederum an die in traditionellen Interpunktionslehren häufiger vorgetragene Forderung, eine gewisse Sparsamkeit beim Umgang mit Ausrufezeichen walten zu lassen. Sehr dezidiert wird dies etwa im DDR-Duden formuliert: „Ein alter Grammatiker nennt das Ausrufezeichen 'Lärmstange'; in einer neueren Stilkunde heißt es 'Punkt im Affekt'. Man setze deshalb kein Ausrufezeichen, wo ein Punkt genügt! Unbegründete Häufung schwächt das Zeichen in seiner Wirkung ab" (Duden 1969: 666).

\footnotetext{
${ }^{23} \mathrm{Zu}$ Gottscheds Interpunktionslehre vgl. auch Höchli (1981: 190-198) sowie Rinas (2014: 145, 153-158).

${ }^{24}$ Der Text der Gundling-Ausgabe ist in Fraktur gedruckt. Die hier kursivierten Stellen sind im Original in Antiqua gesetzt.
} 
Es ließen sich noch weitere Parallelen zwischen den Diskussionen des Smiley-Gebrauchs und der (frühen) Interpunktionslehre ziehen:

- Wie schon in der Einleitung am Beispiel von The Unofficial Smiley Dictionary ausgeführt wurde, gibt es teilweise Bemühungen, eine hierarchische Stufenfolge der Smileys zu definieren. Ähnliche Hierarchisierungen spielten auch in der Interpunktionslehre eine wichtige Rolle. Schon die ursprüngliche Gliederung in Periode, Colon und Comma ist im Kern hierarchisch angelegt (vgl. Abschnitt 4.1). In der frühen Neuzeit wurde dieser Ansatz weiter geführt; insbesondere Aldus Manutius d. J. hat in seinem 1566 veröffentlichten Werk Orthographiae ratio die hierarchische Gliederung der Zeichen nach ihrer „Stärke” herausgearbeitet (vgl. Bieling 1880: 23; Müller 1882: 294f.; Parkes 1993: 49).

- Ein Bestandteil der Interpunktionstheorie ist die Graphotaktik, d. h. die Lehre von der Kombinierbarkeit und den Abfolgeregularitäten der Interpunktionszeichen. Hierzu gehören beispielsweise Feststellungen wie die, dass nach dem Komma ein Leerzeichen steht, vor dem Komma hingegen nicht, oder dass Ausrufezeichen iterieren können (!!!!), Semikolons aber nicht (*;;;) (vgl. Bredel (2011: 4) und das dortige Kapitel 3). Wie in Abschnitt 5 gezeigt wurde, wird auch die Graphotaktik der Smileys diskutiert, ${ }^{25}$ wobei sich deutliche Parallelen zur Interpunktionstheorie zeigen, sei es dass die Frage der (Nicht-)Setzung von Leerzeichen bei Smileys behandelt wird (vgl. Abschnitt 5, iii-c), sei es dass auf die Möglichkeit der Iterierung innerhalb von Emoticons eingegangen wird (welche wiederum der Verstärkung und hierarchischen Ordnung von Emoticons dienen kann) (vgl. etwa Wirth 2005: 81; Siever 2006: 79).

- Haase et al. (1997: 65) sehen ein wesentliches Merkmal der Smileys darin, dass sie Ideogramme seien, die „im Sinne konzeptioneller Mündlichkeit” eingesetzt würden und somit eine gewisse Affinität zur gesprochenen Sprache aufwiesen. Damit ist die Frage aufgeworfen, inwieweit sich die typischen medialen Kontexte des SmileyGebrauchs (Chats, E-Mails, SMS-Nachrichten) der gesprochenen Sprache zuweisen lassen, eine Frage, die intensiver diskutiert wurde (vgl. etwa Runkehl/Schlobinski/Siever 1998: 63; Wilde 2002; Hess-Lüttich/Wilde 2003; Dürscheid 2003: 13f., 2011). Interessant hieran ist, dass auch die traditionelle Interpunktion sich im Ausgang von Reflexionen über die mündliche Realisierung von Äußerungen entwickelt und sich erst später ein "abstrakterer" Gebrauch etabliert hat (vgl. Abschnitt 4.1; vgl. auch 2.2).

Derlei Parallelen nähren die Vermutung, dass die Smileys ein Interpunktionssystem in statu nascendi konstituieren, welches diverse Gemeinsamkeiten mit den älteren Interpunktionskonzeptionen aufweist. Allerdings ist hier ein wichtiger Unterschied zu berücksichtigen: Während die traditionelle Interpunktion sich praktisch ,konkurrenzlos“ entwickelt hat, muss sich das Smiley-System zwangsläufig in Auseinandersetzung mit dem elaborierten traditionellen Interpunktionssystem entfalten.

Bislang fristet die „Smiley-Interpunktion“ eher ein Nischendasein; sie kommt vor allem in SMS- und Chat-Kommunikation sowie in E-Mails zur Anwendung. Die Verbreitung gerade

\footnotetext{
${ }^{25}$ In Bezug auf Smileys wird die Diskussion allerdings bislang offenbar fast ausschließlich unter Laien geführt.
} 
in diesen Textsorten ist sicher kein Zufall, sondern bestimmten medialen Bedingungen geschuldet: Namentlich die SMS- und Chat-Kommunikation erfolgt nicht nur zeitnah, sondern unterliegt auch quantitativen Beschränkungen, so dass Smileys hier ein hochwillkommenes Mittel darstellen, mit dem sich Sprecherhaltungen in besonders ökonomischer Weise ausdrücken lassen (vgl. etwa Jasper 1997:48; Crystal 2006: 41f.; Siever 2006: 77f.).

Ob Smileys sich auch in anderen Textgattungen durchsetzen werden, ist bislang weitgehend offen. Dürscheid (2005: 94f.) verweist auf einen Gebrauch durch Schüler auch in der nichtelektronischen Kommunikation, etwa in „Schülerbriefe[n], die unter der Bank weitergereicht werden". Hingegen behauptet etwa Siever (2006: 86), dass Smileys bislang noch kaum in Weblogs gebraucht würden.

Gegenwärtig lässt sich kaum abschätzen, ob der Smiley-Gebrauch sich weiter ausbreiten oder aber stagnieren wird. Aber auch wenn er sich nicht weiter entwickeln oder gar ,aus der Mode kommen" sollte, so ändert das wenig an der obigen Einschätzung, wonach die Smileys Eigenschaften eines frühen Interpunktionssystems aufweisen, bei dem sich erste Grammatikalisierungstendenzen nachweisen lassen. Insofern sind die Smileys zweifellos ein interessantes Phänomen aus schriftlinguistischer Sicht, ein Phänomen, dessen Entwicklung weiter verfolgt und analysiert werden sollte.

\section{Literaturverzeichnis}

Alexander, Thor (2006): Elektronischer Knigge. Netiquette und Verhaltensregeln für die berufliche und private Tele- und Onlinekommunikation. Berlin: Rhombos.

Amtliches Regelwerk (2006) = Deutsche Rechtschreibung. Regeln und Wörterverzeichnis. Entsprechend den Empfehlungen des Rats für deutsche Rechtschreibung. Überarbeitete Fassung des amtlichen Regelwerks 2004. München/Mannheim.

[online: http://rechtschreibrat.ids-mannheim.de/download/regeln2006.pdf]

Antos, Gerd (1996): Laien-Linguistik. Studien zu Sprach- und Kommunikationsproblemen im Alltag. Tübingen: Niemeyer.

Basedow, Johann Bernhard (1756): Lehrbuch prosaischer und poetischer Wohlredenheit. Kopenhagen: Rothensche Buchhandlung.

Baudusch, Renate (1986): Punkt, Punkt, Komma, Strich. Regeln und Zweifelsfälle der deutschen Zeichensetzung. Leipzig: VEB Bibliographisches Institut.

Beißwenger, Michael (2000): Kommunikation in virtuellen Welten: Sprache, Text und Wirklichkeit. Stuttgart: ibidem.

Bieling, Alexander (1880): Das Princip der deutschen Interpunktion nebst einer übersichtlichen Darstellung ihrer Geschichte. Berlin: Weidmann.

Bredel, Ursula (2011): Interpunktion. Heidelberg: Winter.

Bünting, Karl-Dieter/Bergenholtz, Henning (1989): Einführung in die Syntax. 2. Auflage. Frankfurt/M.: Athenäum.

Bussmann, Hadumod (ed.) (2002): Lexikon der Sprachwissenschaft. 3. Auflage. Stuttgart: Kröner.

Crowley, Sharon/Hawhee, Debra (2004): Ancient Rhetorics for contemporary students. 3. Auflage. New York etc.: Pearson - Longman.

Crystal, David (2006): Language and the Internet. 2. Auflage. Cambridge: Cambridge University Press. 
Curtius, Ernst R. (1993[1948]): Europäische Literatur und lateinisches Mittelalter. 11. Auflage. Tübingen/Basel: Francke.

Donatus-Schönberger, Axel (2009): Die Ars maior des Aelius Donatus. Lateinischer Text und kommentierte deutsche Übersetzung. Frankfurt/M.: Valentia.

Drews, L. (1992): „Aposiopese”. In: Ueding, Gert (ed.) (1992): Historisches Wörterbuch der Rhetorik. Bd. 1. Tübingen, Niemeyer: 828-830.

Duden, Konrad (1876): Versuch einer deutschen Interpunktionslehre zum Schulgebrauch. Schleiz: Rosenthal. [Wieder abgedruckt in: Nerius, Dieter (2005): Konrad Dudens orthographische Schriften. Hildesheim/Zürich/New York, Olms: 192-207.]

Duden (1969) = Der Große Duden. Wörterbuch und Leitfaden der deutschen Rechtschreibung. 16. Auflage. Leipzig: VEB Bibliographisches Institut.

Duden (1986) $=$ Duden - Die Rechtschreibung. 19. Auflage. Mannheim/Wien/Zürich: Duden.

Duden $(2000)=$ Duden - Die deutsche Rechtschreibung. 22. Auflage. Mannheim etc.: Duden.

Dürscheid, Christa (2003): „Netzsprache - ein neuer Mythos”. www.ds.unizh.ch/ lehrstuhlduerscheid/docs/netzsprache.pdf [10.02.2014].

Dürscheid, Christa (2005): „E-Mail - verändert sie das Schreiben?” In: Siever/Schlobinski/Runkehl (eds.) (2005): Websprache.net. Sprache und Kommunikation im Internet. Berlin/New York, de Gruyter: 85-97.

Dürscheid, Christa (2006): „Merkmale der E-Mail-Kommunikation”. In: Schlobinski (ed.) (2006): Von *hdl* bis *cul8r*. Sprache und Kommunikation in den Neuen Medien. Mannheim etc.: Duden: 104-117.

Dürscheid, Christa (2011): „Parlando, Mündlichkeit und neue Medien. Anmerkungen aus linguistischer Sicht". Schweizerische Zeitschrift für Bildungswissenschaften 33/2: 175190.

Dürscheid, Christa (2012): Einführung in die Schriftlinguistik. 4. Auflage. Göttingen: Vandenhoeck \& Ruprecht.

ELL2 = Brown, Keith E. (ed.) (2006): Encyclopedia of Language \& Linguistics. 2. Auflage. 14 Bde. Oxford: Elsevier.

Ferretti, Vittorio (2004): Wörterbuch der Elektronik, Datentechnik, Telekommunikation und Medien. Teil 1: Deutsch-Englisch. 3. Auflage. Berlin/Heidelberg: Springer.

Fiehler, Reinhard (2003): „Was sind die Grundeinheiten gesprochener Sprache? Ein altes Problem und ein neuer Lösungsvorschlag”. Sprachtheorie und germanistische Linguistik 13/2: 145-172.

Friedmann, Leonid (1972): „Funktionale Besonderheiten selbständiger Sätze als Absatzkomponenten“. Deutsch als Fremdsprache 9/1972: 298-303.

Fuchs, Walter R. (1968): Knaurs Buch der Denkmaschinen. München/Zürich: Knaur.

Ghersi, Lenny/Sue Lee/Karadagi, Allan (2002): Gabler Kompakt-Lexikon Internet. Wiesbaden: Gabler.

Glück, Helmut (ed.) (2010): Metzler Lexikon Sprache. 4. Auflage. Stuttgart: Metzler.

Gottsched, Johann Christoph (1776): Vollständigere und Neuerläuterte Deutsche Sprachkunst. 6. Auflage. Leipzig: Breitkopf.

Gundling (1734) = Hempel, Christian Friedrich (ed.): D. Nicol. Gundlings vollständige Historie der Gelahrtheit. 1. Bd. Frankfurt/Leipzig: Spring. 
Günther, Hartmut/ Ludwig, Otto (eds.) (1994/1996): Schrift und Schriftlichkeit. 2 Bde. Berlin/New York: de Gruyter.

Haase, Martin et al. (1997): „Internetkommunikation und Sprachwandel”. In: Weingarten Rüdiger (ed.): Sprachwandel durch Computer. Opladen: Westdeutscher Verlag.: 51-85.

Hennig, Mathilde (2006): Grammatik der gesprochenen Sprache in Theorie und Praxis. Kassel: Kassel University Press.

Hess-Lüttich, Ernest/Wilde, Eva (2003): „Der Chat als Textsorte und/oder als Dialogsorte?” Linguistik online 13/1: 161-179.

Heynatz, Johann Friedrich (1782): Die Lehre von der Interpunktion. Zweite, durchgängig verbesserte Ausgabe. Berlin: Mylius.

Heynatz, Johann Friedrich (1803): Deutsche Sprachlehre zum Gebrauch der Schulen. 5. Auflage. Berlin: Mylius.

Höchli, Stefan (1981): Zur Geschichte der Interpunktion im Deutschen. Berlin/New York: de Gruyter.

Hoffmann, Ludger et al. (1992): „Podiumsdiskussion zum Satzbegriff”. In: Hoffmann, Ludger (ed.): Deutsche Syntax. Ansichten und Aussichten. Jahrbuch des Instituts für deutsche Sprache (1991). Berlin, de Gruyter: 363-416.

Jasper, Dirk (1997): Internet Newsgroups. Düsseldorf/München: Econ.

Kjørup, Søren (1997): “Pictograms”. In: Posner, Roland/ Robering, Klaus/Sebeok, Thomas A. (eds.): Semiotik. Ein Handbuch zu den zeichentheoretischen Grundlagen von Natur und Kultur. Bd. 4. Berlin/New York, de Gruyter: 3504-3510.

Klussmann, Niels (1999): Lexikon der Kommunikations- und Informationstechnik. 2. Auflage. Heidelberg: Hüthig. [CD-Rom-Fassung]

Lausberg, Heinrich (1973): Handbuch der literarischen Rhetorik. 2. Auflage. München: Hueber.

Lischka, Konrad (2007): „25 Jahre Seitwärts-Smiley: Ich bin :-)”. Spiegel online www.spiegel.de/netzwelt/web/25-jahre-seitwaerts-smiley-ich-bin-a-498428.html [10.02.2014].

Lutz, Hans-Rudolf (1996): „Moderne Piktographie”. In: Günther/Ludwig (eds.): Schrift und Schriftlichkeit. 2 Bde. Berlin/New York: de Gruyter: 1638-1650.

Mautner, Gerlinde (2011): Wissenschaftliches Englisch. Wien: UVK.

Müller, Beat Louis (1985a): „Geschichte der Satzdefinition”. Zeitschrift für germanistische Linguistik 13/1985: 18-42.

Müller, Beat Louis (1985b): Der Satz. Definition und sprachtheoretischer Status. Tübingen: Niemeyer.

Müller, Johannes (1882): Quellenschriften und Geschichte des deutschsprachlichen Unterrichtes bis zur Mitte des 16. Jahrhunderts. Gotha: Thienemann.

Parkes, Malcolm Beckwith (1993): Pause and Effect. An Introduction to the History of Punctuation in the West. Berkeley/Los Angeles: University of California Press.

Quintilianus: zitiert nach der Ausgabe: Marcus Fabius Quintilianus (2006): Ausbildung des Redners. Zwölf Bücher. Zweiter Teil, Buch VII-XII. Hg. u. übers. v. Helmut Rahn. Darmstadt: Wissenschaftliche Buchgesellschaft.

Riederer, Friedrich (1517): Spiegel der waren Rhetoric. Straßburg. (= VD 16: R 2341)

Ries, John (1931): Was ist ein Satz? Prag: Taussig \& Taussig. 
Rinas, Karsten (2011): Sprache, Stil und starke Sprüche. Bastian Sick und seine Kritiker. Darmstadt: Wissenschaftliche Buchgesellschaft.

Rinas, Karsten (2012): „Zur Geschichte der deutschen Interpunktionslehre vom 15. bis zum 17. Jahrhundert". Sprachwissenschaft 37/1: 17-64.

Rinas, Karsten (2014): „Von der Rhetorik zur Syntax: Die deutsche Interpunktionslehre im Zeitalter der Aufklärung". Sprachwissenschaft 39/2: 115-181.

Rinas, Karsten (2015): „Zum linguistischen Status des Absatzes”. In: Bergerová, Hana/Schuppener, Georg/Szatmár, Petra (eds): Text und Stil im Wandel - neue Perspektiven der Textlinguistik und Stilistik. Wien, Prasensverlag: 139-158. [= Aussiger Beiträge 9].

Runkehl, Jens/Schlobinski, Peter/Siever, Torsten (1998): Sprache und Kommunikation im Internet. Opladen: Westdeutscher Verlag.

Sanderson, David (1993): Smileys. Sebastopol/CA: O'Reilly.

Sanderson, David (1997): „Lexikon der Emotikons”. Zeitschrift für Semiotik 19/3: 307-315.

Scaglione, Aldo (1981): Komponierte Prosa von der Antike bis zur Gegenwart. Bd. 1. Die Theorie der Textkomposition in den klassischen und den westeuropäischen Sprachen. Stuttgart: Klett.

Schlobinski, Peter (2001): Rezension zu: Crystal, David 2001: Language and the Internet. Cambridge.

www.mediensprache.net/en/literatur/rezensionen/docs/1452.pdf [25.02.2016].

Schlobinski, Peter (ed.) (2006): Von *hdl* bis *cul8r*. Sprache und Kommunikation in den Neuen Medien. Mannheim etc.: Duden.

Schmidt, Gurly (2000): „Chat-Kommunikation im Internet - eine kommunikative Gattung?” In: Thimm (ed.) (2000): Soziales im Netz. Sprache, Beziehungen und Kommunikationskulturen im Internet. Opladen, Westdeutscher Verlag: 109-130.

Schmitthenner, Friedrich Jakob (1824): Die Lehre von der Satzzeichnung oder Interpunction in der teutschen Sprache. Frankfurt: Hermann.

Seidel, Eugen (1935): Geschichte und Kritik der wichtigsten Satzdefinitionen. Jena: Frommann.

Siever, Torsten/Schlobinski, Peter/ Runkehl, Jens (eds.) (2005): Websprache.net. Sprache und Kommunikation im Internet. Berlin/New York: de Gruyter.

Siever, Torsten (2006): „Sprachökonomie in den 'Neuen Medien””. In: Schlobinski (ed.) (2006): Von *hdl* bis *cul8r*. Sprache und Kommunikation in den Neuen Medien. Mannheim etc., Duden: 71-88.

Sjurts, Insa (2006): Gabler Kompakt-Lexikon Medien. Wiesbaden: Gabler.

Staab, Gregor (2009): „Satzlehre im Rahmen der klassischen Rhetorik”. In: Fix, Ulla/Gardt, Andreas/Knape, Joachim (eds.): Rhetorik und Stilistik. 2. Halbband. Berlin/New York, de Gruyter: 1498-1514.

Stolt, Birgit (1990): „Redeglieder, Informationseinheiten: Cola und Commata in Luthers Syntax". In: Betten, Anne (ed.): Neuere Forschungen zur historischen Syntax des Deutschen. Tübingen, Niemeyer: 379-392.

Storrer, Angelika (2001): „Getippte Gespräche oder dialogische Texte? Zur kommunikationstheoretischen Einordnung der Chat-Kommunikation". In: Lehr, Andrea et al. (eds.): Sprache im Alltag. Beiträge zu neuen Perspektiven in der Linguistik. Berlin etc., de Gruyter: 439-465. 
Thimm, Caja (ed.) (2000): Soziales im Netz. Sprache, Beziehungen und Kommunikationskulturen im Internet. Opladen: Westdeutscher Verlag.

Trautsch, Christian/Wu, Yixin (2012): „Die Als-ob-Struktur von Emotikons im WWW und in anderen Medien”. Image. Zeitschrift für interdisziplinäre Bildwissenschaft 16/2012: 47-60.

Weingarten, Rüdiger (ed.) (1997): Sprachwandel durch Computer. Opladen: Westdeutscher Verlag.

Weise, Christian (1691): Curiöse Gedancken Von Deutschen Brieffen. Dresden: Mieth.

whatis?com Encyclopedia (2002) = The whatis?com Encyclopedia of Technology Terms Indinanapolis: Que Publishing.

Wilde, Eva (2002): Zwischen Mündlichkeit und Schriftlichkeit: Die Chat-Kommunikation aus linguistischer Sicht. Seminararbeit, Universität Bern. www.chat-bibliography.de/ papers/wilde.pdf [09.02.2016]. (= Bibliography on Chat Communication. Papers \& Studies 1).

Wirth, Uwe (2005): „Chatten. Plaudern mit anderen Mitteln”. In: Siever/Schlobinski/Runkehl (ed.) (2005): Websprache.net. Sprache und Kommunikation im Internet. Berlin/New York, de Gruyter: 67-84.

Wirth, Uwe (2006): „Chatten online”. In: Schlobinski (ed.) (2006): Von *hdl* bis *cul8r*. Sprache und Kommunikation in den Neuen Medien. Mannheim etc., Duden: 118-132.

\section{Internetquellen}

Das-Smiley-Problem. www.civforum.de/showthread.php?27086-Das-Smiley-Problem\&high light=Smiley+Satzzeichen [22.04.2014].

Einfügen von Smileys. http://office.microsoft.com/de-de/excel-help/einfugen-von-smileysHA001119608.aspx [08.04.2014].

Gibt es eine Smileyregelung in der Rechtschreibung? www.forum-3dcenter.org/ vbulletin/archive/index.php/t-305727.html [12.04.2014].

haben-smiley-eine-satzzeichen-funktion. www.nickles.de/forum/off-topic/2007/haben-smileyeine-satzzeichen-funktion-538200052.html [24.04.2014].

NETiquette - deine Etikette im Netz. http://magazin.chringles.de/netiquette-deine-etikette-imnetz/ [02.05.2014].

Netiquette: Smileys vermeiden und die Wortwahl abwägen. www.teltarif.de/arch/2006 /kw47/s23897.html [04.05.2014].

Netiquette beim E-Mailing. http://pub.ids-mannheim.de/abgeschlossen/orbis/net/archiv/ schwichtenberg.html [02.05.2014].

Orig-Smiley. www.cs.cmu.edu/ sef/Orig-Smiley.htm [06.04.2014].

Smilies-und-Satzzeichen. www.civforum.de/showthread.php?44822-Smilies-und-Satzzeichen $\& s=742811094748 \mathrm{~d} 0 \mathrm{~d} 52232 \mathrm{e} 6 \mathrm{bbbfaf9f01} \mathrm{[09.04.2014].}$

Tradition oder rote Trikots? http://forum.football-thugs.net/hooligans-deutschland/wm-2006deutschland-25/tradition-oder-rote-trikots-2271/index2.html [04.04.2014].

The Unofficial Smiley Dictionary. https://w2.eff.org/Net_culture/Net_info/EFF_Net_Guide/ EEGTTI_HTML/eeg_286.html[08.04.2014].

Wikipedia: Emoticon. http://de.wikipedia.org/wiki/Emoticon [02.04.2014].

Wikipedia: Piktogramm. http://de.wikipedia.org/wiki/Piktogramm [02.04.2014].

Wikipedia: Smiley. http://de.wikipedia.org/wiki/Smiley [02.04.2014]. 